\title{
Extents of Generic Prescribing in Hospitals and Community Pharmacies for Diabetic and Hypertensive Outpatients in Eastern Region of Saudi Arabia
}

\author{
Promise Madu Emeka*, Anas Al Ahmed \\ Department of Pharmaceutical Sciences, College of Clinical Pharmacy, King Faisal University, Al Ahsa, KINGDOM OF SAUDI ARABIA.
}

\begin{abstract}
Objective: As population increase, Healthcare resources are stretched and the need to reduce prescription drug spending becomes obvious. The aim of this study was to evaluate the levels of generic prescribing in place of their branded counterparts among type 2 diabetic and hypertensive outpatients in Eastern region of Saudi Arabia. Method: A cross section of 13 Hospitals and 17 Community Pharmacies located in Al Ahsa, Dhahran, Khobar and Dammam (both Government and private) were visited. Out of which 58 Physicians and Pharmacists were interviewed with a structured questionnaire. Reasons for prescribing and serving either generic or branded were also ascertained. $\mathrm{Pa}$ tients' prescriptions were also examined at the various hospital and community pharmacies. Our focus was on prescription drugs for type 2 diabetic and hypertensive patients only and the study lasted for 6 months. Results: Findings revealed that only $26.9 \%$ generic medications were prescribed for both type 2 diabetic and hypertensive patients in the 13 hospitals sampled. It was also observed that while Government hospital prescribed $33.3 \%$ generics, private hospitals had $28.6 \%$ for same patient category. In the Community Pharmacies, only $23.5 \%$ generic were served for diabetics and $11.8 \%$ for hypertensive
\end{abstract}

patients. Overall Hospitals branded prescription were $73.1 \%$ whereas in community Pharmacies, they represented $82.4 \%$ served medications. Conclusion: Levels of generic medication usage appeared to be significantly low. Majority of the Physicians and Pharmacists were of the belief that branded medications give better outcome to patients and this attitude could potentially render Government policies on the use of generic medication ineffective.

Key words: Branded medication, Clinical equivalence, Community Pharmacies, Generics, Hospitals, Physicians.

Correspondence :

Promise Madu Emeka

Department of Pharmaceutical Sciences, College of Clinical Pharmacy,

King Faisal University, Al Ahsa, SAUDI ARABIA.

Phone no: +966503239033

Email: pemeka@kfu.edu.sa

DOI: 10.5530/jyp.2017.9.55

\section{INTRODUCTION}

Prevention and treatment of chronic diseases as well as cost control is the cornerstone in patients' care. Diabetes mellitus and hypertension are costly chronic diseases that have accounted for added economic burden on already stretched health care budgets across the continents. In the UK, $10 \%$ of the NHS budget is expanded on the treatment of type 2 diabetes. ${ }^{1}$ The cost of same treatment to the US economy in one year was $\$ 174$ billion. $^{2}$ Whereas in China, the Government's introduction of affordable essential drugs for the control of hypertension in their 2009 health reforms were cost saving measures. ${ }^{3}$ Giving the current worldwide economic downturn, the use of generic medications can further reduce overhead costs for most National Healthcare Providers and this trend is expected to continue in the near future. ${ }^{4}$ Development of generic medications is based on the pharmacological properties of the innovator brands, which connotes proof of bioequivalence in terms of standard kinetic profile, efficacy and safety in healthy subjects..$^{5-7}$ There are two questions prescribers and other healthcare professionals are asking regarding generic medications;

1. Is therapeutic equivalent guaranteed in generic substitutions?

2. Can simple bioequivalence be enough to guarantee comparable clinical efficacy and safety?

These questions arise in that generics may differ in many cases, from branded medications in their excipient contents, which could potentially exhibit different absorption rates and consequently affect their bioavailability. Consequently, the approval of a generic medication by Drug Regulatory Authorities, like Saudi Food and Drug Authority (SFDA) suggests that it works the same way as its branded equivalent. Therefore, generic prescriptions should not be looked at as if compromising quality of treatment. Systemic reviews have documented clinical equivalence between generic and branded cardiovascular medications. ${ }^{5,8}$ Hence, the use of generics will not only be a lifesaver but also will reduce high cost of care. ${ }^{8}$ Despite all these, Physicians, Pharmacists and patients still harbour doubt about the efficacy of generic medications. ${ }^{9}$ According to US Food and Drug Administration (FDA) ${ }^{10}$ generics are drug products that are comparable to branded/reference ones in dosage form, strength, route of administration, quality, performance characteristics and intended use. While it is true that Physicians and Pharmacists behaviour are key factors that can motivate patients to accept generic medications, ${ }^{11}$ it is equally true that their non-acceptance of all-generic substitution promotes negative attitudes towards their use. The Saudi Government has mandated its Healthcare Institutions to embrace the idea of generic substitutions and hence its usage. The question therefore is, has the fear of prescribing generics abated? Are there real changes in behaviour, attitude and perceptions of the physicians and pharmacists despite numerous interventions highlighted to address it? ${ }^{12,13}$ If factors influencing the prescriptions of branded over generic medications in Saudi Arabia have been addressed, what is the degree of positive response to this policy in terms of extent of generic prescriptions in its hospitals? There are no reports on the levels or extent of generic prescriptions in the hospitals and other healthcare centres in the Eastern region. The aim of the present study therefore, was to assess the levels of generics prescribing for 
T2DM and hypertensive patients in Government/private hospitals and community Pharmacies within the region.

\section{MATERIALS AND METHOD}

\section{Protocol}

The present study was conducted using a modified interview-questionnaire survey format, adopted from Kumar et al. ${ }^{14}$ A preliminary test was done with 5 physicians and 5 pharmacists at the King Faisal University polyclinic, to assess the questionnaire's validity and reproducibility. On each occasion, the definitions of generic and brand medications according to FDA were read to each respondent during the interview. This was to ensure that both the respondents and the authors have same interpretations and to gather informed views regarding the level of generic prescribing for T2DM and hypertensive patients. In addition, socio-demographic data of the physicians/pharmacists, including their practice experience, volume of daily prescriptions and number of generic medications prescribed and served in their hospital/community pharmacies. Their knowledge regarding bioequivalence, beliefs regarding quality, safety and efficacy of generic medications and cost in comparison to branded products. The purpose and importance of the study was explained and participation voluntary and information obtained was treated confidentially and kept anonymous. The College of Clinical Pharmacy ethical committee endorsed this study, which was in line with Deanship of Scientific Research (DSR), King Faisal University ethical requirements for conducting such studies.

\section{Questionnaire administration}

A cross section of physicians in Government and private hospitals located in Al Ahsa, Dhahran, Khobar and Dammam cities of Saudi Arabia were approached for this evaluation. The study was conducted using face-to-face interview-questionnaire. Selection criteria included, those who have more than 1 year experience in treating chronic diseases, specifically T2DM and hypertension (HTN). They include specialist or family health physicians working in Government and private hospitals. Also community Pharmacies within same location were also part of the study. The study was conducted for 6 months in 13 Hospitals and 17 community pharmacy outlets within the kingdom randomly selected.

\section{Statistical analysis}

Data obtained were analysed using SPSS software version 19. They were subjected to frequency analysis, T-tests and Fisher Exact chi-square tests to check association between the groups. Statistical significance was indicated at $\mathrm{p}<0.05$.

\section{RESULTS}

General prescription patterns for T2DM and HTN observed from this study revealed that in both Government and private hospitals, levels of utilization of generic medications were significantly $(p<0.05)$ in favour of branded ones. Generics prescription utilization for T2DM and HTN during the period under review was $26.9 \%$ as against $73.1 \%$ for branded medications. This observation is represented in Figure 1, and is a combined reflection of extent of usage in both government and private hospitals.

Figure 2 shows the comparisons between prescription patterns in chronic diseases treatments in Government and private hospitals. Results indicate that in both hospitals, generic drugs prescriptions for T2DM and HTN were both significantly $(\mathrm{p}<0.05)$ low and appear to be in preference to branded medications. Using Fisher Exact $\mathrm{Chi}^{2}$ to compare both hospitals' use of either generic or branded medications, there appeared to be no significant difference, as they seemed to be similar in what they are doing.

In Table 1, the details of medications prescribed for the treatment of T2DM and HTN in both Government and private hospitals are displayed. From the data, metformin represented $33.3 \%$ by generic and $53.3 \%$ brand. T-tests analysis showed that P-values did not indicate any statistical difference between generics and brand forms of oral hypoglycaemics. However, for HTN treatment, the use of branded amlodipine represents a significant figure $(\mathrm{p}<0.05)$. The result also showed that glibenclimide, gliclazide and atenolol were prescribed as brands.

Comparison of prescription patterns for T2DM and HTN in both Government and private hospitals are shown in Figure 3. The results show a preference for branded medications over their generic counterparts. The study found that $61.5 \%$ represents branded medications for T2DM and $76.1 \%$ for HTN. These observations appeared to be statistically significant $(\mathrm{p}<0.05)$ when you compare treatments for T2DM using generics and branded oral hypoglycaemics. In addition, the same trend plays out for the treatments of HTN by both Government and private hospitals (Figure 3).

As shown in Figure 4, community Pharmacists served 23.5\% generic medications for the treatment of T2DM and $76.5 \%$ branded ones during the same period under review. The same trend followed the medications prescribed for HTN treatments as they served $88.2 \%$ branded drugs versus $11.8 \%$ generics. In both cases, serving of branded medications represented a significant $(\mathrm{p}<0.05)$ attitude.

\section{DISCUSSION}

The promotion of generics utilization and hence its substitution can be best described as an economic strategy aimed at cost containment in the area of drug usage. In Saudi Arabia, Healthcare services are free and the burden of chronic diseases (especially T2DM and HTN) on its budget has increased over the years. ${ }^{15}$ Therefore, increasing generic medication usage will provide a cheaper alternative to the healthcare budget burden. However, this study is reporting an overwhelming preference for branded forms of metformin and amlodipine in the treatment of T2DM and HTN at its hospitals located in the eastern region. Our report is comparable to the findings of that found in Sharjah hospitals UAE, in which $19.4 \%$ was the level of generic prescriptions while in India, reports showed $19.8 \%{ }^{16,17}$ In Nigeria, Okonta et $a l^{18}$ reported $38.7 \%$ generic prescriptions in their tertiary hospitals. Contrary to these aforementioned reports, other parts of the world are showing higher levels of generic prescriptions usage in their hospitals. Example is the District hospitals Thailand, which showed $73.9 \%$ overall generic prescriptions for all the treatments. ${ }^{19}$ According to other reports, England had $68.9 \% 5$ years ago while Canada showed $54 \%$ of all prescriptions dispensed, as generic medications in 2009. ${ }^{20}$ Drug procurement by hospitals depend on the physicians' request and they hold the key for enhancing the use of generics as well. ${ }^{19,21}$ Furthermore, our study revealed only $38.3 \%$ generic prescriptions for T2DM treatments and $23.9 \%$ for HTN in both government and private hospitals. Similar trends were described in the study conducted in India, where levels of generic prescriptions in a tertiary hospital for T2DM treatment represented only $25.8 \% .^{22}$ In another study on the levels of generic prescriptions in Lombardy hospitals, Italy, results showed $36 \%$ for HTN treatments. ${ }^{23}$ Still on the treatment of HTN, another study showed $28.2 \%$ generic prescriptions, which was not significantly different from our own findings. ${ }^{24}$ These come against the backdrop of many reports indicating that Physicians and Pharmacists held many positive and favourable views in terms of generic medications utilization. If these positives are properly channelled, they will motivate hospitals and patients in accepting generic medications. ${ }^{11}$ Moreover, documented evidence showed that Physicians hold the key to enhancing the use 
Table 1: Detailed Drug Prescription Pattern in Government and Private Hospitals for the Treatment of Type 2 Diabetes Mellitus and Hypertension

\begin{tabular}{cccc}
\hline & Generic (\%) & Branded (\%) & P-Value \\
Type 2 Diabetes Mellitus & & & \\
Metformin & 33.3 & 53.3 & $0.03^{*}$ \\
Glibenclimide & 0 & 6.7 & N.D \\
Gliclazide & 0 & 6.7 & N.D \\
Hypertension & & & \\
Amlodipine & 7.7 & 53.8 & $0.001^{*}$ \\
Atenolol & 0 & 15.4 & ND \\
ACEIs & 7.7 & & 0.10
\end{tabular}

${ }^{*}$ Indicates $\mathrm{P}$ value $\leq 0.05$ and is considered statistical significant. $\mathrm{ND}=$ Not determined

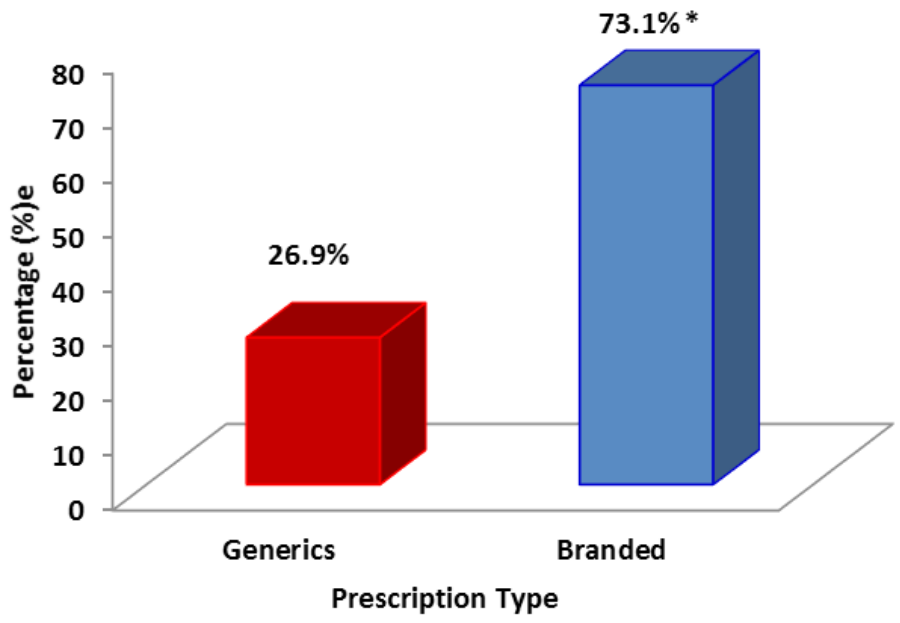

Figure 1: Prescription Pattern for both type 2 diabetes mellitus and hypertensive Patients in Government and Private Hospitals.

*Indicates $P$ value $\leq \mathbf{0 . 0 5}$ and is considered statistical significant for the general use of branded medication over generics.

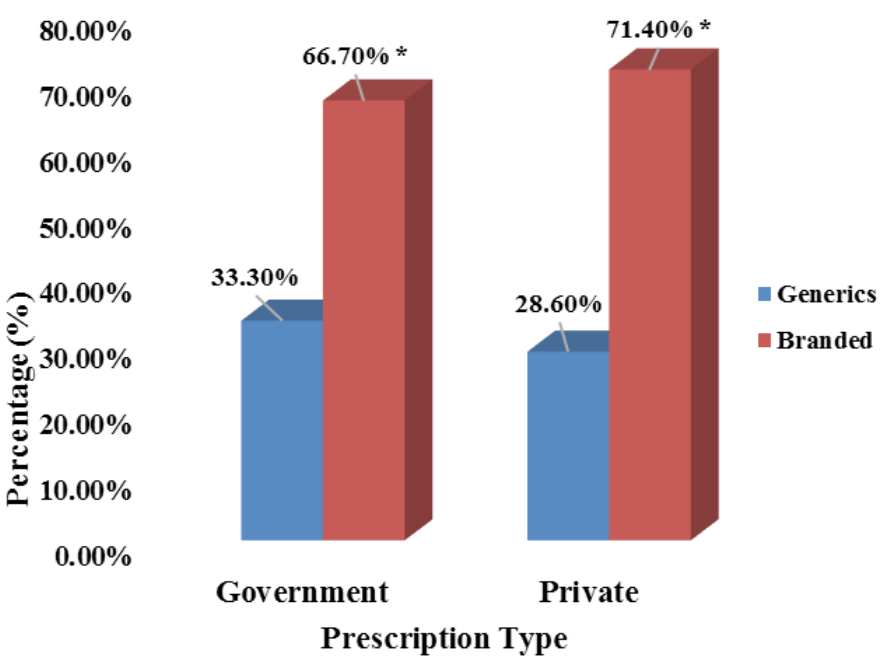

Figure 2: Comparison of prescription patterns between Government and private hospitals for patients with T2DM and HTN.

*Indicates $P$ value $\leq \mathbf{0 . 0 5}$ and is considered statistical significant for the use of branded medication over generics for each of the hospital.

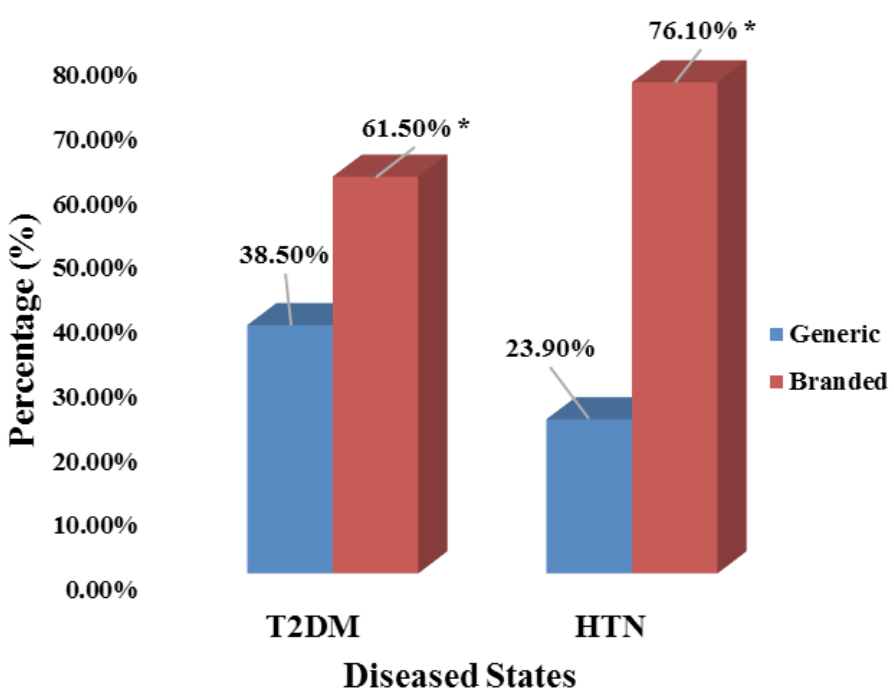

Figure 3: Prescription Patterns comparison betweenT2DM and HTN by Government and Private Hospitals.

*Indicates $P$ value $\leq \mathbf{0 . 0 5}$ and is considered statistical significant for the use of branded in the treatment of chronic diseases.

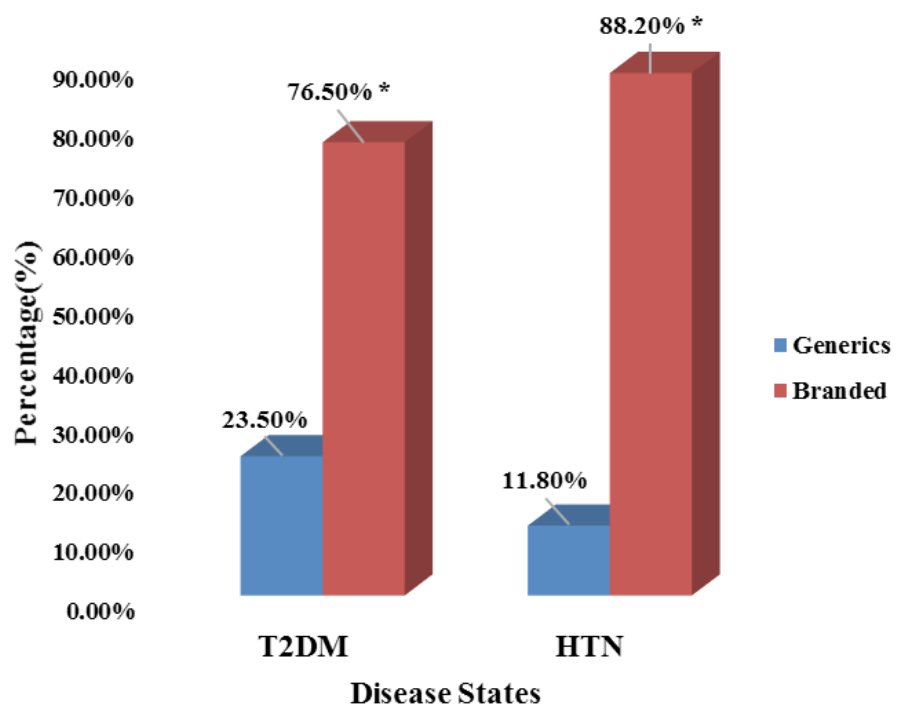

Figure 4: Medications served by Community Pharmacies to Patients with T2DM and HTN.

*Indicates $P$ value $\leq 0.05$ and is considered statistical significant considering that less generic medication is served.

of generic medications. ${ }^{21}$ Their attitudes reflect questionable differences in this generic versus branded saga and is due mainly to what they call observed variance in therapeutic outcomes. This indicates that there are still, perceived doubts regarding the efficacy, quality and clinical equivalence of generic medications. Physicians and pharmacists agree that sometimes, there could a genuine reason to use branded medications but this should be as situations demand. ${ }^{25,26}$ in their report agreed that the use of generic medications does not always guarantee cost savings for certain diseases, even though clinical outcome might be similar to their branded counterparts. On the other hand, community Pharmacies have been known to consider gain over affordability when dispensing medications to patients. ${ }^{13}$ We report here that the levels of generic medications served in the community pharmacies where significantly in favour of branded medications. This finding could be a reflection of the society's attitude towards generic medications, as Saudis prefer European or branded med- 
icines. ${ }^{27}$ The trend follows true for T2DM and HTN drug treatments. In the community pharmacies, options are available since they stock both generic and branded drugs but in the hospital pharmacies, the situation is different. According to a recent evidence, hospital pharmacists are generally influenced by physicians' decision on branded medications, only engaging in generic substitutions when drugs prescribed are not available. ${ }^{13}$ In a situation where branded medications are prescribed, the pharmacists are under obligation to serve it even if the equivalent generic is in stock. The use of generics allows flexibility in both dispensing and procurement. These alternatives are regarded as safe and effective as their branded counterparts. ${ }^{28}$ Notwithstanding, the questions physicians are asking could explain their reluctance to whole-heartedly embrace generic prescribing, implying that they trust the branded medications more. ${ }^{29}$ This obviously reflects in the extent of the generics prescribing in their hospitals. There could be variations in products size, appearance and packaging, despite universal Good Manufacturing Practice regulations. However, if generic medications registration are approved and granted license for use, it then follows that guarantee is given in terms of equal clinical equivalence and safety with the branded counterparts. We therefore, suggest a vigorous enlightenment campaign by the regulatory authority, aimed at Physicians, Community Pharmacists and patients, to address this anomaly.

\section{CONCLUSION}

From the foregoing, the levels of generics prescribed in both Government and private hospitals for the treatment of T2DM and HTN diseases were low. Majority of the Physicians and Pharmacists are of belief that branded medications give better outcomes. Their attitudes could potentially render Government policies on the use of generic medications unsuccessful. In other to promote more utilization of generics, attention should be paid to the physicians' attitudes and observations. Government could still reduce healthcare bills by advocating increased use of generic alternatives were possible, in its hospitals.

\section{ACKNOWLEDGEMENT}

Authors hereby express their appreciation to Saudi FDA for granting the authors knowledgeable insight on their approval procedure for generic medications in the Kingdom. We also wish to thank the Deanship for Scientific Research, King Faisal University for approving this study.

\section{CONFLICT OF INTEREST}

Authors do hereby declare on conflict of interest, and this study was not funded.

\section{ABBREVIATION USED}

T2DM: Type 2 diabetes mellitus; HTN: Hypertension; FDA: Food and Drug Administration; SPSS: Statistical Package for the Social Sciences.

\section{REFERENCES}

1. Tao L, Wilson ECF, Wareham NJ, Sandbæk A, Rutten GEHM, Lauritzen T, et al. Cost-effectiveness of intensive multifactorial treatment compared with routine care for individuals with screen-detected Type 2 diabetes: analysis of the ADDITION-UK cluster-randomized controlled trial. Diabet Med. 2015;32(7):907-19.

2. American Diabetes Association (ADA). Economic costs of diabetes in the U.S. In 2007. Diabetes Care. 2008;31(3):596-615.

3. Gu D, He J, Coxson PG, RasmussenPW, Huang C, Thanataveerat A, et al. The Cost-Effectiveness of Low-Cost Essential Antihypertensive Medicines for $\mathrm{Hy}$ pertension Control in China: A Modelling Study. Goldhaber-Fiebert JD, ed. PLoS Medicine. 2015;12(8):e1001860.
4. Caldeira D, Fernandes RM, Costa J, David C, Sampaio C, Ferreira JJ. Branded Versus Generic Clopidogrel in Cardiovascular Diseases: A Systematic Review. J Cardiovasc Pharmaco. 2013;61(4):277-82.

5. Vetchy D, Vetcha M, Rabiskova M, Gryczova E, Bartosikova L. Comparison in vitro felodipine release rate from the original versus generic product with controlled release of the drug. Medicina (Kaunas). 2007;43(4):326-31.

6. Sicras-Mainar A, Navarro-Artieda R. Physicians' and patients' opinions on the use of generic drugs. J Pharmacol Pharmacother. 2012;3:268-70.

7. Gasser UE, Fischer A, Timmermans JP, Arnet I. Pharmaceutical quality of seven generic Levodopa/Benserazide products compared with original Madopar® / Prolopa®. BMC Pharmacol Toxicol. 2013;14(1):24.

8. Shrank WH, Choudhry NK, Liberman JN, Brennan TA. The use of generic drugs in prevention of chronic disease is far more cost-effective than thought, and may save money. Health Aff (Millwood) 2011;30(7):1351-7.

9. Shrank WH, Liberman JN, Fischer MA, Girdish C, Brennan TA, Choudhry NK. Physician perceptions about generic drugs. Ann Pharmacother. 2011;45(1):31-8.

10. US Food and Drug Administration (FDA). Approval of generic drugs. In: Generic drugs. Silver Spring, MD: FDA; 2008.

11. Kobayashi E, Karigome H, Sakurada T, Satoh N, Ueda S. Patients' attitudes towards generic drug substitution in Japan. Health Policy 2011; 99:60-5.

12. Alghasham AA. Generic drug prescribing in central Saudi Arabia: Perceptions and attitudes of physicians. Ann Saudi Med. 2009;29(1):24-9.

13. Albadr $Y$, Khan TM. Factors influencing community pharmacist decision to dispense generic or branded medicines; Eastern Province, Alahsa, Saudi Arabia. Saudi Pharm J. 2015;23(2):143-6.

14. Kumar R, Hassali MA, Saleem F, Alrasheedy AA, Kaur N, Wong ZY, et al. Knowledge and perceptions of physicians from private medical centres towards generic medicines: a nationwide survey from Malaysia. J Pharm Policy Pract. 2015;8(1):11

15. Mokdad AH, Tuffaha M, Hanlon M, El Bcheraoui C, Daoud F, Al Saeedi M, et al. (2015) Cost of Diabetes in the Kingdom of Saudi Arabia, 2014. J Diabetes Metab 6:575. doi:10.4172/2155-6156.1000575.

16. Sharif SI, Alabdouli AH, Sharif RS. Drug Prescribing Trends in a General Hospital in Sharjah, United Arab Emirates." Am J Pharmacol Sci. 2013;1(1):6-9.

17. Khan AN, Kaur A, Khar RK, Khanam S. Medicine Prescribing Preference and Patient Adherence: Perspectives of Indian Medical Practitioners. J Young Pharm. 2015;7(4s):446-54

18. Okonta JM, Nduka SO, Idodo VE. Prescribing Pattern of Antihypertensive and Antidiabetic Agents in a Secondary Healthcare Institution in Nigeria. J Pharm Sci Res. 2013;5(1):12-17.

19. Plianbangchang $P$, Jetiyanon $K$, Suttaloung $C$, Khumchuen L. Physicians' generic drug prescribing behavior in district hospitals: a case of Phitsanulok, Thailand. Pharm Pract (Granada). 2010;8(3):167-72.

20. El-Dahiyat FA, Kayyali R. Community pharmacists' perceptions towards generic medicines and their opinions on future generic substitution policy implementation: A descriptive study from Jordan. J Generic Med. 2013;10(2):97-104.

21. Dunne SS. What Do Users of Generic Medicines Think of Them? A Systematic Review of Consumers' and Patients' Perceptions of, and Experiences with, Generic Medicines. Patient 2016 May 3. [Epub ahead of print] DOI: 10.1007/ s40271-016-0176-X.

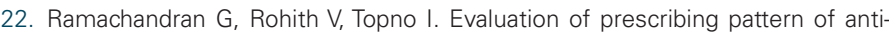
diabetic drugs using WHO prescribing indicators in a tertiary care hospital in Puducherry: A cross-sectional study. Pharma Innovation. 2015;4(5):76-80.

23. Corrao G1, Soranna D, La Vecchia C, Catapano A, Agabiti-Rosei E, Gensini G, et al. Medication persistence and the use of generic and brandname blood pressure-lowering agents. J Hypertens. 2014;32(5):1146-53.

24. Nachiya RAMJ, Parimalakrishnan S, Rao M R. Study on drug utilization pattern of antihypertensive medications on out-patients and inpatients in a tertiary care teaching hospital: A cross sectional Study. Afr J Pharm Pharmacol. 2015;11: 383-96.

25. Gallelli L, Maida F, Staltari O, Rende P, Russo E, Caroleo B, et al. Recurrence of atrial fibrillation after switching from brand to generic atenolol. J Pharmaco Pharmacother. 2015;6(1):39-41.

26. Duh MS, Cahill KE, Paradis PE, Cremieux PY, Greenberg PE. The economic implications of generic substitution of antiepileptic drugs: a review of recent evidence. Expert Opin Pharmacother. 2009;10(14):2317-28.

27. Sarah A. (2013). Saudis prefer Western-made medicines Retrieved $16^{\text {th }}$ Sept 2013, from http://www.arabnews.com/news/454252.

28. Eze UIH, Odunayo OO. Evaluation of drug use among diabetic hypertensive patients in a teaching hospital. Int J Drug Dev Res. 2010;2(4):703-10.

29. Gallelli L, Palleria C, De Vuono A, Mumoli L, Vasapollo P, Piro B, et al. Safety and efficacy of generic drugs with respect to brand formulation. J Pharmacol Pharmacother. 2013;4(Suppl S1):110-4. 